\title{
Emotive Qualities of Parametrically Designed and 3D Printed Surfaces
}

\author{
Ok, Jeongbin ${ }^{a} \&$ Scudder, Daniel $^{\mathrm{b}}$ \\ ${ }^{a}$ School of Design, Victoria University of Wellington, New Zealand. jeongbin.ok@vuw.ac.nz \\ ${ }^{\mathrm{b}} \mathrm{School}$ of Design, Victoria University of Wellington, New Zealand. dan.scudder@gmail.com
}

\begin{abstract}
Surfaces play a crucial role in design. Constituting a physical and visual interface of an object, a surface not only reveals important information about the identity of the entity it encloses but also determines impressions, evaluation, and expectations we have about the object. Despite their significance, surfaces have long been considered to be a subordinate part of form rather than an independent design element, especially under the reign of mass production. This paper challenges the conventional hierarchy and demonstrates a systematic and customisable process of creating purpose-specific, context-oriented surfaces which provide their own set of form and function.
\end{abstract}

Various digital technologies, including parametric modelling, geometric alteration, and $3 D$ printing, were employed as main tools and thoroughly utilised across creation, modification and fabrication of surfaces. Through experimentation with software and mechanical configurations, a series of high-resolution surfaces with different parameters were produced. Furthermore, the potential for direct digital manufacturing (DDM) and its practical penetration was investigated by producing all surfaces directly from a $3 D$ printer without the use of post-production processes, which provided an understanding of the restrictions and opportunities of the technology.

User testing was carried out over participants who observed and interacted through touch with each surface. With each interaction the user was asked to populate a questionnaire form that asks them to identify their interpretation of the surface on a spectrum. The questions were devised to explore three distinct areas of inquiry geometry, physical properties and emotive responses. The results were then analysed using the method of design of experiments (DOE) in order to identify parameters that are responsible for arousing specific visual, tactile, and emotional qualities and explore how these surfaces can be interpreted emotively, physiologically and aesthetically by the user.

Keywords: Surface, Parametric, Digital, Design, 3D printing 


\section{Introduction}

The surface of a designed object is our first point of contact that forms our first impressions of the object. In particular the interaction with the tactile qualities of the surface create the initial sense-data that we use to attribute value and desirability to reassure what we see (Jansson-Boyd, 2011). From childhood touching and holding an object is an important part of acquiring and structuring haptic knowledge that forms later experiences and expectations (Piaget, 1952). Beyond being a sense that we use to experience objects, touch is also a sense that is instinctive. When we touch a warm, moist and smooth surface we often have the response of disgust as an instinctive response to detect and avoid pathogens that could be passed onto us. (Oum et. al., 2011). Such an instinctive response is indicative of a catalogue of hard-wired responses that designers can use to communicate with the user through surfaces. Yet due to materials and surfaces often being a consequence to the manufacturing process rather than a deliberate decision there has been little research conducted within the field of design on pinpointing these responses.

Mass manufacturing has created a hierarchy within the design process when it comes to material selection. Due to this hierarchy the surface of the material is subordinate to the processes used to form that material. For example, when machining aluminium the mill can only access the surface of the metal and onto this apply surface roughness. The finer the surface roughness the more time it takes and therefore more associated cost. It is possible to machine small details onto the surface but this will inflate the cost as the tooling will need to be replaced more frequently. This balance of detail and cost the process has over the past decade been circumvented with the introduction of 3D printers that are able to print the finished product directly. Direct digital manufacturing (DDM) is a natural progression of additive manufacturing (AM) that allows designers and engineers to create complex forms through CAD and have them printed ready for use directly from the machine. DDM works in stark contrast to the economies of scale that drives mass manufacturing in that it allows detailed and complex forms to be produced without the tradeoff between detail and cost (Wohler, 2009). Already we see within the grassroots maker movement inventors' 3D printing complex components and on-selling these as finished products. As the resolution for consumer 3D printers increases and are able to be printed directly the surfaces of these objects is no longer over shadowed by the hierarchy imposed by mass manufacturing.

Alongside the ability to directly digitally manufacture products is the ability to form the surfaces of the objects parametrically. Parametric design is the ability to drive the form or surface of a product with an algorithm or set of instructions that respond to a set of values that define the parameters of the design. Parametric design is used within architecture, a style called parametricism, to create the designs for buildings that are responsive and dynamic as opposed to the traditional regular format that is often attributed to architecture. A style exemplified by architects such as Zaha Hadid, Frank Gehry and Greg Lynn. The combination of parametric design and DDM represent the ability to create objects that are responsive, sometimes purpose built to a specific user at no extra cost. The potential for parametric design with 3D printed surfaces is that a designed object will be able to have different surfaces that respond to user depending where they interact with the object. The design potential for being able to illicit certain responses for example temperature, direction or pleasure would represent a shift in what the designer would be able communicate to the user and how. As well as this the attention to the surface of the object will give a much needed sense of value between $3 \mathrm{D}$ printed objects, between a quickly printed part and a much considered and highly designed object.

3D printers are diverse in their range and applications though can be divided into three main groups, selective laser sintering (SLS), stereolithography (SLA) and filament fused fabrication (FFF) each with their own strengths and weaknesses. SLS printing is achieved by pushing a fine layer of powder across and using a laser to melt and merge the powder particles together. While this process affords the greatest 
versatility in materials in that it can fuse both plastics and metals it is also the most prohibitive as it requires specific material compositions and equipment to extract the parts from the powder. SLS is used as a DDM process and used for aeronautical and automotive applications. SLA a process that is able to reach the greatest resolution as it prints by using a photosensitive resin that hardens when light bonds the molecules together in a process called photopolymerisation. The drawback of SLA is that it must use specific resins to produce the models and this relegates this process to prototyping and rarely are the finished parts functional. Some SLA processes require time consuming cleaning of the parts after completion. FFF is the most accessible of the 3D printing processes as it is the simplest. A small nozzle heats up a filament of what is typically a plastic and extrudes the melted plastic layer by layer to create a form. FFF in terms of materials is the most versatile of the methods and is able to print in rubber, wood, plastics and most materials that are able to be heated and extruded. This also makes it the cheapest and is themes commonly seen consumer model 3D printer. FFF lacks in resolution compared to the other processes though with advancements in the resolution of the motors that drive the extruder head greater resolution is able to be achieved. Another restriction of the FFF printer is that it is the only methods that must compete with gravity. Support structures are often constructed as part of the printing process but are generally undesirable. Each of these methods are becoming widely available and are already becoming common place within universities and industry alike.

As 3D printers decrease in price they will become more viable for more producers. Eventually we will see 3D printed products more frequently within our daily lives but our current approach to 3D printing by focusing solely on form creates the potential for homogeny of surfaces. Surfaces of 3D printed objects are currently defined by the process used. For example, with SLS creating a granular surface and FFF creating easily identifiable ridges from the layering. These default surfaces for these processes, while currently novel, will eventually become homogenous. Like the association created between smooth surfaces of injection moulded plastics and plastic toys (Karana et. al. 2008) so too will associations be constructed with 3D printing. This presents a problem for designers looking to make use of the versatility of the process but unable to create designs that have surfaces that can be differentiated from every other 3D printed object. Yet consumer 3D printers now feature motors that are able to create a layer resolution of 50 microns meaning that there is an opportunity to create previously unobtainable fine geometries to create intentional surfaces.

While the area of 3D printed parametric design is relatively new there is much interest in the potential of its applications. Notably architect Neri Oxman of MIT is undertaking research into the potential of growing materials and structures through $3 \mathrm{D}$ printing that respond to load as opposed to rigid preformed materials used for construction (Oxman; Rosenburg, 2007). Her Carpal Skin (2010) design is a prospective design to explore the use of 3D printing in the creation of biomimicry surfaces that can be utilised to treat patients who suffer from carpal tunnel syndrome. Oxman's work to date is a good example of how surfaces can be driven through algorithms and the visual outcomes and complexity that is possible.

This paper explores the relationship between computer generated parametric surfaces and the interpreted emotive responses from the participants. This is a short but necessary explorative study that will give direction for future research that will delve deeper into the surfaces that elicited consistent results. This initial systematic research will create a frame work for what surfaces are possible to print, at what scale and start to give meaning to the digital surfaces that are being created. The future application of these considered surfaces onto products has the intention of adding emotive value to objects that are created using a method that treats each print alike. Furthermore, this research looks to bring more focus on a new realm of design opportunity and the possibilities that are presented when designers are not restricted by mass manufacturing methods to apply surfaces to their design. 


\section{Methods}

\subsection{Software}

Many different geometries and printing methods were explored to discover what surfaces were possible to print. Through the explorations it was found that printing the details of a surface was accompanied by a different set of difficulties than that experienced when focusing solely on form. From this initial foray into the design of parametric surfaces we found that most the common 3D modelling software such as Solidworks and Rhino were ill-equipped to process the small details applied onto a surface. Instead an open source 3D modelling program OpenSCAD was found the most successful in creating digitally created surfaces as it is driven by code as opposed to a graphical user interface (GUI).

\subsection{Hardware}

For this research three FFF printers were tested for suitability, the Micro 3D (M3D), 3D Printing Systems Up Box and Up Mini. The UP Box featured fast operation, temperature controlled cabinet for optimal layer adhesion during the print and heated platform for the model adhesion to the platform. While larger prints were of the best quality once the scale of the surfaces decreased we experienced issues with clogging of the nozzle making surface features below $2 \mathrm{~mm}$ unobtainable. The UP Mini is a smaller version of the UP Box and featured heated platform but experienced the same issues as the UP Box with smaller details. Both the UP Box and UP Mini have a layer resolution of 100 microns and both required use of their proprietary software which didn't allow for the uploading of custom G-Code (the set of instructions used to control the $3 \mathrm{D}$ printer to create the $3 \mathrm{D}$ print). For the exploration of printing parametric geometries, we used a consumer M3D printer that was able to move along the Z-axis and lay each layer 50 microns apart, currently the higher end of resolution available for FFF printers. The M3D was an inexpensive printer that while allowing for greater detail also lacked the features such as heated platform, enclosed cabinet for temperature control and fast operation that would have allowed for consistent results. The M3D however was preferable as it allowed for importing of custom G-Code from slicer software Cura, which is a program that will read 3D models and slice it into layers and create the GCode to run the 3D printer. This greater control allowed us to adjust finer setting to create prints that were otherwise unachievable on the UP Box or UP Mini and of surprising detail. Through testing we were able to decrease the layer resolution to 25 microns which substantially increased the print time but did not produce discernible results.

\subsection{D Printing}

We considered each of the three main processes for 3D printing SLS, FFF and SLA when engaging with the exploration process. When selecting the process to focus on we had a set of four criteria. 1) Ability to produce parts that were ready for use straight from the printer meaning no post-processes were necessary. 2) Wide range of readily available materials for wider range of experimentation. 3) The ability to upload our own custom G-Code in order to pursue different paths of enquiry. 4) The ability to produce fine detailed surfaces that have structural integrity that allow them to be handled after printing. Of the three methods we settled on FFF as these printers are readily available and not cost prohibitive. They have a wide range of filaments to choose from of different materials and some are able to produce strong fine details with no need for post processing from the printer. As these machines are able to be purchased we have been able to acquire a 3D printer that allows the uploading of custom G-Code. FFF machines however must work against gravity and in our experiments we had to create geometries that allowed us to print without the use of support material that would have made the surface details redundant. SLS printers are large and expensive machines that are frequently used as a service and due to their cost they would not have allowed for the uploading of custom G-Code. Also the process which allows for standard sized 
3D models to be printed with relative ease is also brittle when the details go below $1 \mathrm{~mm}$. In addition to this the SLS method requires specially formed powders for sintering though they SLS method does allow for metals to be printed. Finally, the SLA method which is affordable and is able to produce parts in the finest detail requires a special photo sensitive resin restricting materiality. Some SLA printers also require time consuming removal of support material which with very fine and delicate surface features made the process unfeasible.

\subsection{Parameters}

The parameters used to generate the specimens used for this study were the result of an exploratory study into which geometries are able to be produced at high resolution of 50 microns along the Z-axis on a FFF 3D printer. Three sets of successful geometries were used from our initial explorations. 1) Inverted spheroids were used as they provided the greatest detail in our explorations and were able retain the sharpest form. The inverted spheroids were used to test the tactile qualities of pointed structures and were merged along the hemisphere so that when they were scaled along either axis they would produce a pillar between each iteration. The spheroids were able to be deformed along either axis and also the proximity between each feature could be adjusted. The inverted spheroids were used to test harsh pointed surfaces. 2) Spheroids were used to compliment the inverted spheroids and operated under the same set of parameters. They created rounded features that that sought to elicit less harsh tactile qualities. They were also merged along the hemisphere for consistency through scale transformation. 3) Iterative boxes were the final geometry to be tested due to the lines they produced when set alongside each other. Of the parameters were two sets riy and rux that would slightly rotate each iteration with the angle compounding. Additionally, the parameter $m i$ was used to control the frequency of the iterations. If the value was lowered the iterations would occur more frequently. This allowed for strong linear forms to be produced to test whether strong direction within the surface had an effect on participants.

\subsection{Survey}

The survey that was undertaken was to gather initial data to give direction for future 3D printing experiments to create more targeted surfaces. Our initial undertaking was primarily based upon the work of Elvin Karana who has developed a method for gathering data that attributes meaning to materials. The survey Karana et. al. had developed asked the participants to find high quality materials and then ill out a survey with a set of questions that related to a range of sensorial properties such as elasticity, roughness and weight but required no direct contact with specific materials (Karana et. al., 2009). A similar study that is based upon the same work of Karana did require participants to directly touch samples and fill out a survey form that contained texture lexicons (Hope et. al., 2013). Our survey blended the sensorial and lexicon aspect of both aforementioned surveys. Our survey spanned three main categories of responses, physiological such as temperature, geometric such as perceived complexity of the surface and emotive based response such as attractive or traditional. Participant were asked to touch each sample and then fill out the form depending on the response they felt. Each form contained questions and also allowed the participant to describe the surface in their own way and to rate how much they personally liked the surface, this was repeated over all 16 samples (Table 1). At the end of the survey the participants were asked to fill out a final sheet which asked them to choose their favourite and least favourite sample and explain why. (Fig. 1) 


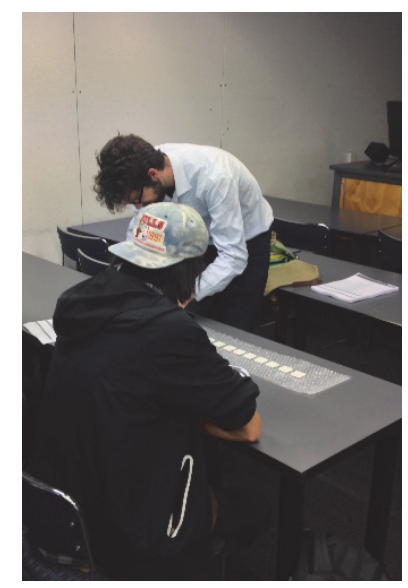

Fig. 1 A typical survey setting

Table 1. Emotional qualities on a 8-point scale

\begin{tabular}{|c|c|c|c|c|}
\hline 1. & [Smooth] & 12345678 & [Rough] & (SR) \\
\hline 2. & [Sharp] & 12345678 & [Blunt] & (SB) \\
\hline 3. & [Hard] & 12345678 & [Soft $]$ & (HS) \\
\hline 4. & [Warm] & 12345678 & {$[\mathrm{Cool}]$} & (WC) \\
\hline 5. & [Fragile] & 12345678 & [Sturdy] & (FS) \\
\hline 6. & [Simple] & 12345678 & [Complex] & (SC) \\
\hline 7. & [Valuable] & 12345678 & [Cheap] & (VC) \\
\hline 8. & [Natural] & 12345678 & [Synthetic] & (NS) \\
\hline 9. & [Casual] & 12345678 & [Formal] & $(\mathrm{CF})$ \\
\hline 10. & [Pleasant] & 12345678 & [Unpleasant] & (PU) \\
\hline 11. & [Modern] & 12345678 & [Traditional] & (MT) \\
\hline 12. & [Attractive] & 12345678 & [Unattractive] & $(\mathrm{AU})$ \\
\hline
\end{tabular}

\section{Results and Discussion}

Fig 2 shows names and definitions of parameters used in this research. $r$ refers to the radius of spheroid features, and $s x$ and $s y$ indicate scale transformation of spheroid along the $\mathrm{X}$ - and $\mathrm{Y}$ - axis, respectively. For set 3, $f x, f y$, and $f z$ represent dimensions of box features, and $p x$ and $p y$ are used to show distance between two adjacent box features along the X-and Y-axis, respectively. Rotation of a box feature along the X- and Y-axis, respectively, in degrees is expressed using riy and rux, and $m i$ is an indicator that shows how many iteration steps are in a unit length: 0.5 means 2 times as many features exist, respectively. 


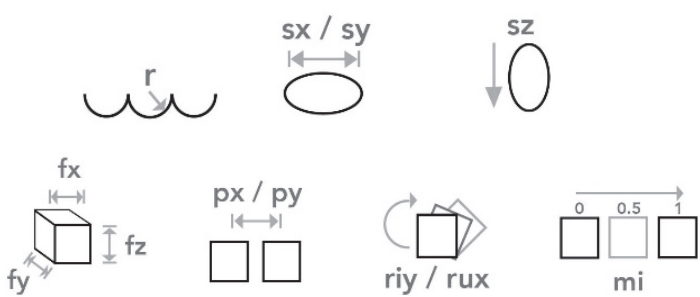

Fig 2. Geometircal definitions of parameters

Tables 2, 3, and 4 detail surface profiles belong to the three sets and combinations of parameters responsible for each of the profiles. Fig 3, 4, and 5 depict visualisations of the surface profiles.

Table 2. Parameters and their combinations used for set 1

\begin{tabular}{cccc}
\hline Surface & $\boldsymbol{r}$ & $\boldsymbol{s y}$ & $\boldsymbol{s z}$ \\
\hline $1-2$ & 5.00 & 4.00 & 2.00 \\
$1-3$ & 2.75 & 2.50 & 1.25 \\
$1-4$ & 5.00 & 1.00 & 0.50
\end{tabular}

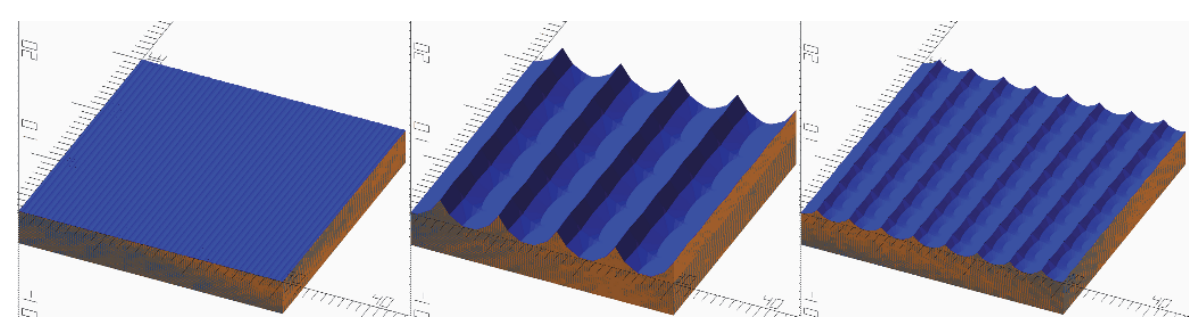

Fig 3. Visualisations of surfaces belong to set 1

Table 3. Parameters their combinations used for set 2

\begin{tabular}{cccc}
\hline Surface & $\boldsymbol{r}$ & $\boldsymbol{s y}$ & $\boldsymbol{s z}$ \\
\hline $2-1$ & 2.75 & 2.50 & 1.25 \\
$2-2$ & 0.50 & 4.00 & 0.50 \\
$2-3$ & 5.00 & 4.00 & 2.00 \\
$2-4$ & 5.00 & 1.00 & 0.50 \\
$2-5$ & 0.50 & 1.00 & 2.00 \\
\hline
\end{tabular}




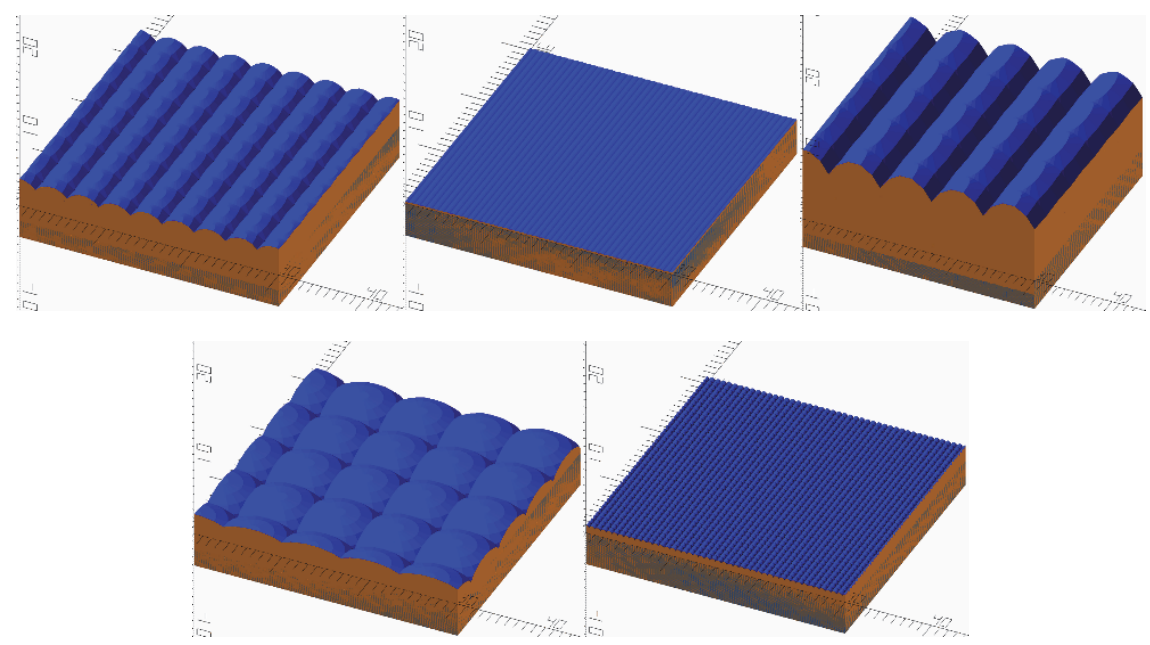

Fig. 4 Visualisations of surfaces belong to set 2

Table 4. Parameters their combinations used for set 3

\begin{tabular}{ccccccc}
\hline Surface & $\boldsymbol{f y}$ & $\boldsymbol{f} \boldsymbol{z}$ & $\boldsymbol{p y}$ & $\boldsymbol{r i y}$ & $\boldsymbol{r u x}$ & $\boldsymbol{m i}$ \\
\hline $3-1$ & 0.50 & 4.00 & 1.00 & 0.00 & 45.00 & 0.20 \\
$3-2$ & 2.00 & 0.50 & 1.00 & 0.00 & 0.00 & 1.00 \\
$3-3$ & 2.00 & 4.00 & 4.00 & 20.00 & 45.00 & 1.00 \\
$3-4$ & 2.00 & 0.50 & 4.00 & 0.00 & 45.00 & 0.20 \\
$3-5$ & 1.25 & 2.25 & 2.50 & 10.00 & 22.50 & 0.60 \\
$3-6$ & 2.00 & 4.00 & 1.00 & 20.00 & 0.00 & 0.20 \\
$3-7$ & 0.50 & 0.50 & 4.00 & 20.00 & 0.00 & 0.20 \\
$3-8$ & 0.50 & 4.00 & 4.00 & 0.00 & 0.00 & 1.00 \\
$3-9$ & 0.50 & 0.50 & 1.00 & 20.00 & 45.00 & 1.00 \\
\hline
\end{tabular}




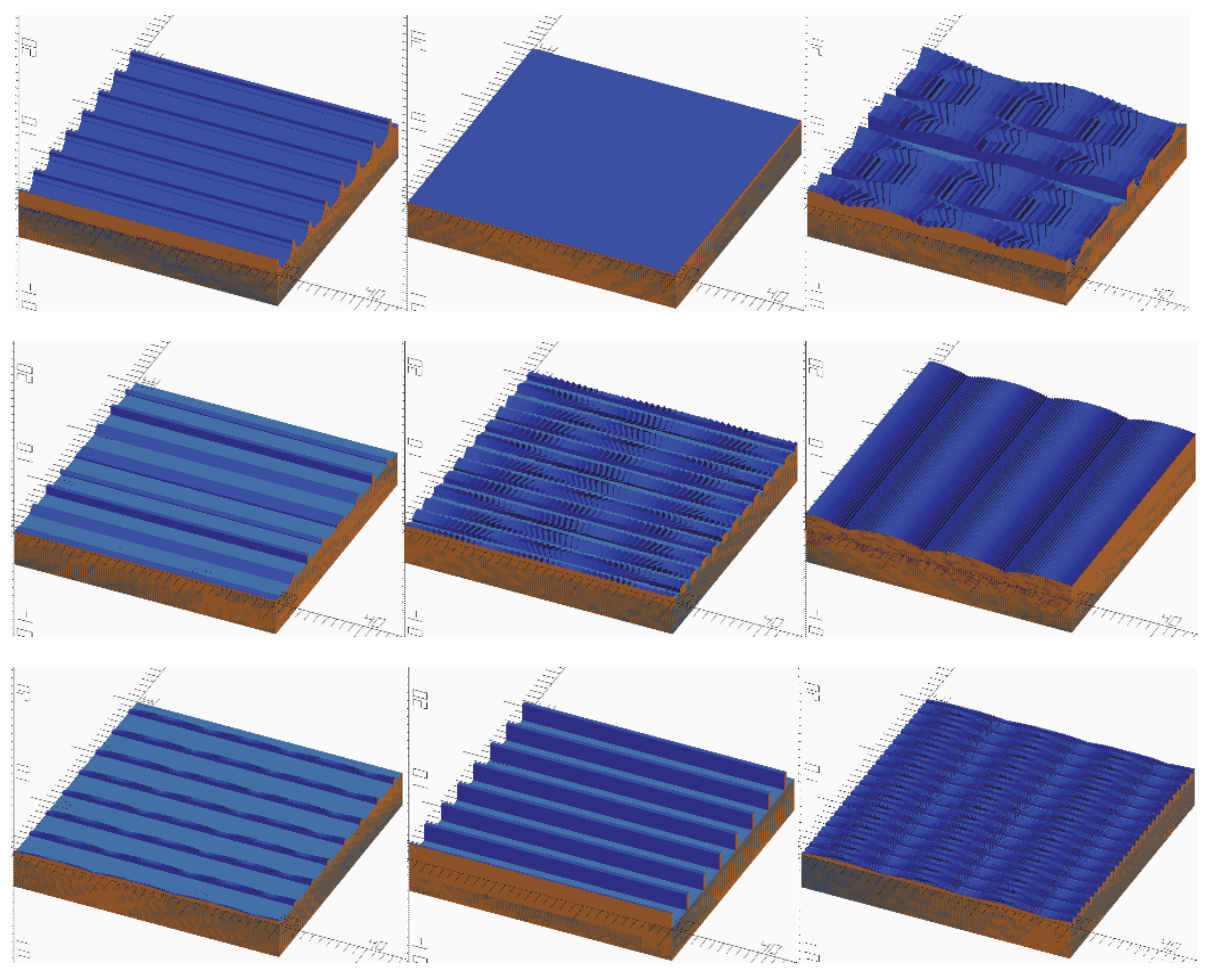

Fig 5. Visualisations of surfaces belong to set 3

The collected questionnaires were coded by assigning a number between 1 and 8 to each of the corresponding answers on the scale and the numerical values were rearranged by emotional quality. This conversion enabled statistical examination to determine whether each of the parameters has significant effect on the responses at $95 \%$ confidence level $(\mathrm{p}=0.05)$ by using two-way factorial analysis that processes the parameters as factors. Tables 5, 6, and 7 detail parameters across the three sets which have statistically significant effects. Interactions between two or among three parameters were observed in sets 2 and 3.

Table 5. Parameters that have significant effects (set 1)

\begin{tabular}{cccc}
\hline Emotional quality scale & A & B & $\mathbf{C}$ \\
\hline SR & V & V & - \\
SB & V & - & - \\
HS & - & - & - \\
WC & - & - & - \\
FS & - & - & - \\
SC & - & V & - \\
VC & - & - & - \\
NS & - & - & - \\
CF & - & - & - \\
PU & V & - & - \\
MT & - & - & - \\
AU & - & - & - \\
\hline
\end{tabular}


Emotive qualities of parametrically designed and $3 D$ printed surfaces.

Table 6. Parameters that have significant effects (set 2)

\begin{tabular}{|c|c|c|c|c|}
\hline Emotional quality scale & $\mathbf{A}$ & B & $\mathbf{C}$ & $\mathbf{I}$ \\
\hline SR & $\mathrm{V}$ & - & $\mathrm{V}$ & $\mathrm{V}$ \\
\hline SB & $\mathrm{V}$ & - & - & - \\
\hline HS & - & - & - & - \\
\hline WC & $\mathrm{V}$ & - & - & - \\
\hline FS & $\mathrm{V}$ & - & - & - \\
\hline $\mathrm{SC}$ & $\mathrm{V}$ & - & - & - \\
\hline $\mathrm{VC}$ & - & - & - & - \\
\hline NS & - & - & $\mathrm{V}$ & - \\
\hline $\mathrm{CF}$ & $\mathrm{V}$ & V & - & V \\
\hline PU & - & - & V & - \\
\hline MT & - & - & - & V \\
\hline $\mathrm{AU}$ & - & - & $\mathrm{V}$ & - \\
\hline
\end{tabular}

Table 7. Parameters that have significant effects (set 3)

\begin{tabular}{cc}
\hline Emotional quality scale & Interactions \\
\hline SR & C E A AF \\
SB & C E A AF B \\
HS & C AF E F D \\
WC & C E AF D \\
FS & A D C \\
SC & E C D AF \\
VC & D \\
NS & C A \\
CF & C A \\
PU & C D E A F \\
MT & E \\
AU & C D \\
\hline
\end{tabular}

$(\mathrm{A}=f y, \mathrm{~B}=f z, \mathrm{C}=p y, \mathrm{D}=r i y, \mathrm{E}=r u x, \mathrm{~F}=m i)$

The subsequent analysis focused on the parameters identified as statistically significant. Fig 6 shows effects of the three parameters of set 1 on the SR scale, and the relationship depicted on the left suggests that the larger radius of spheroid $(r)$ on the surface is, the rougher the surface feels, which is intuitively 
understandable. The graph on the right in the meawhile represents that a surface consists of elongated geometries (high $s y$ ), such as ovoids, compared to perfect circles, is more likely to be felt smoother. Such geometries are also responsible for making surfaces feel complex on the SC scale, which aligns well with everyday observations (Fig 7).

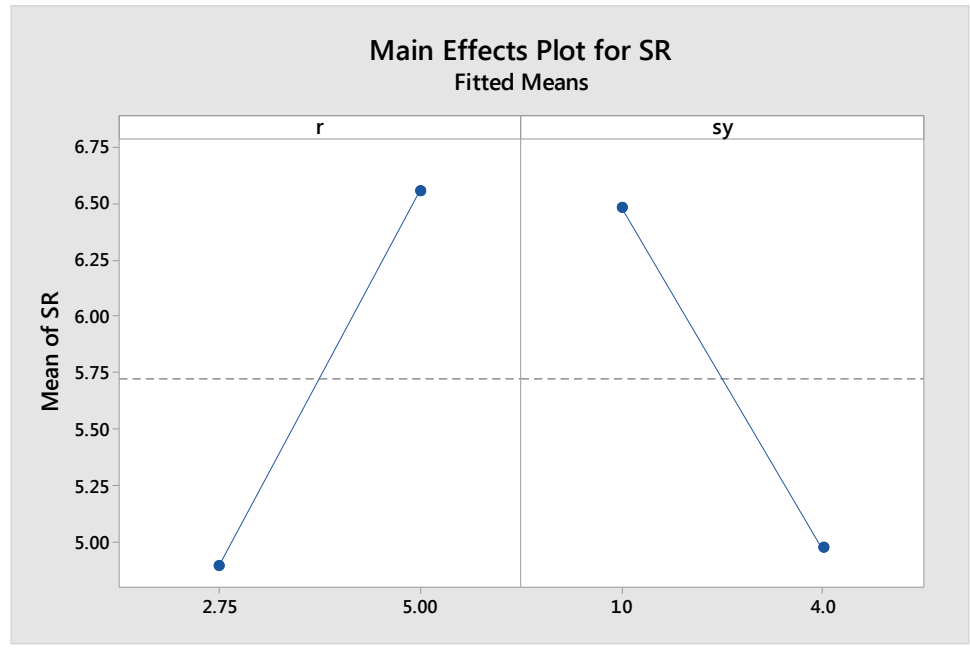

Fig 6. Effects plot for SR (set 1)

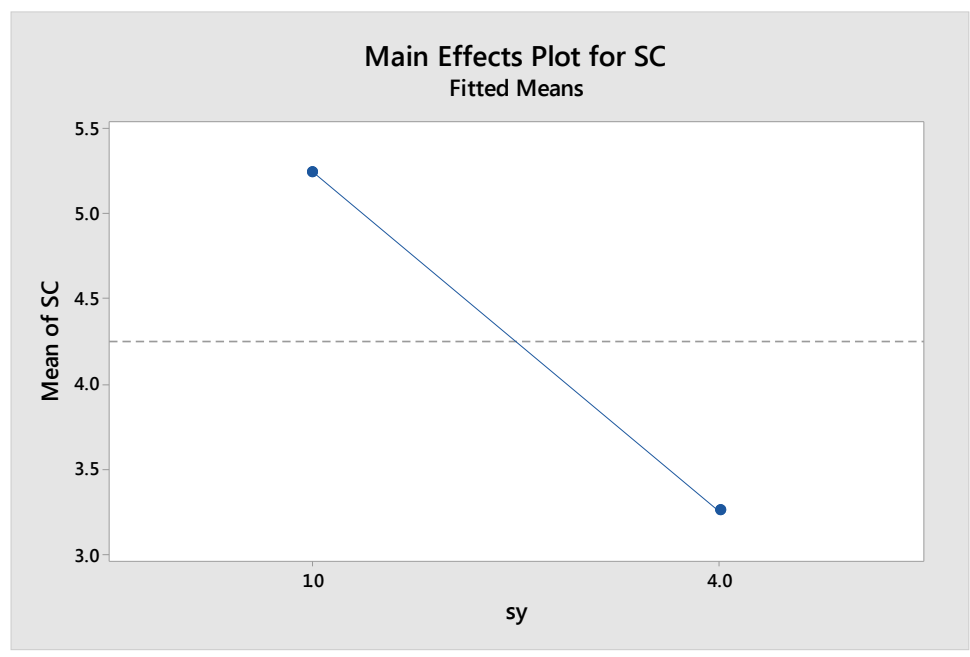

Fig 7. Effects plot for SC (set 1)

On the SB scale, as depicted on Fig $8, r$ is the only significant parameter. Low $r$ values, which dictate densely packed small patterns in a unit area, are linked to surfaces that feel sharper. However, such surfaces can be seen quite unattractive at the same time (Fig 9). 


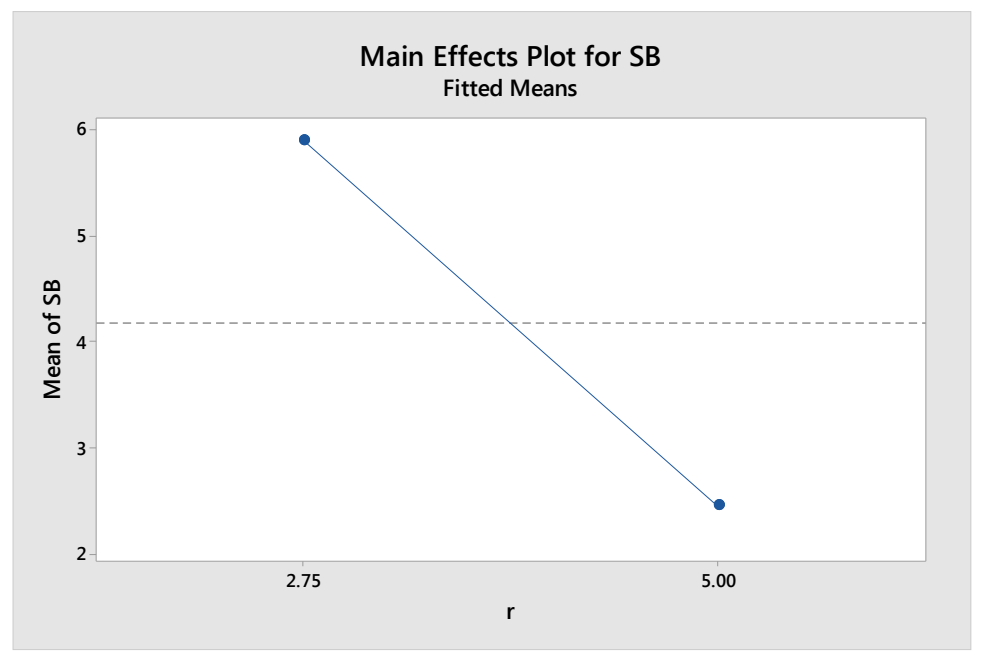

Fig 8. Effects plot for SB (set 1)

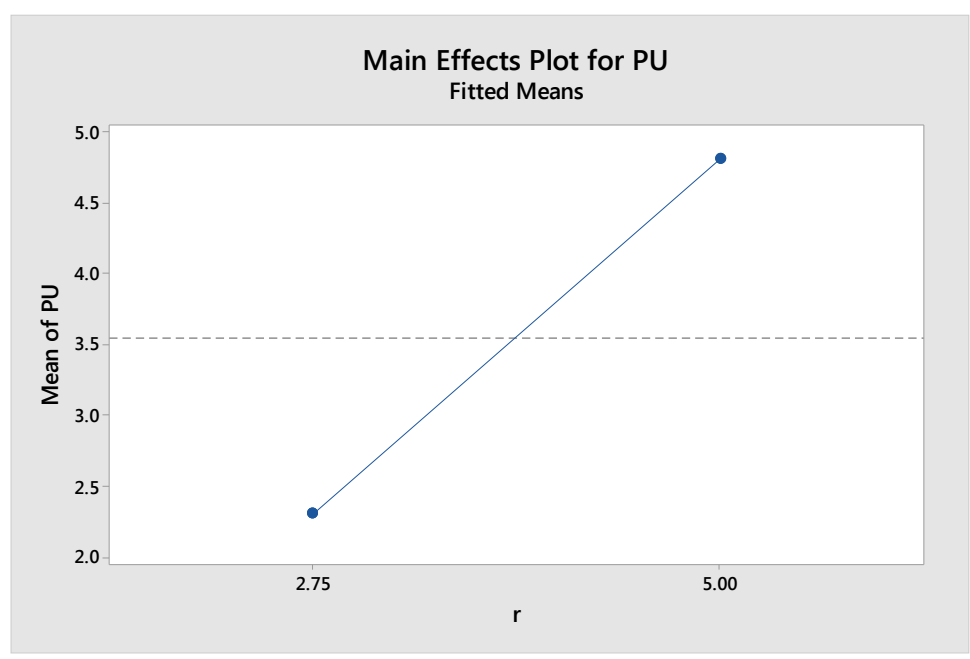

Fig 9. Effects plot for $P U$ (set 1)

Set 2 includes surfaces that are basically inverse of the ones belong to set 1 . Fig 10 clearly demonstrates why two parameters $r$ and $s z$, with the exception of $s y$ which has marginal effect, are flagged as having significant effects on the SR scale. While increased radius $r$ makes the surface smoother (left), oblong patterns produced as a result of high $s z$ values contribute to surfaces that feel rough (right). This phenomenon which responses are influenced by two or more parameters at the same time, either synergistically or attenuatively, is called interaction, and in this case it suggests an important possibility of creating highly smooth or rough-feeling surfaces by combining more than one parameters. 


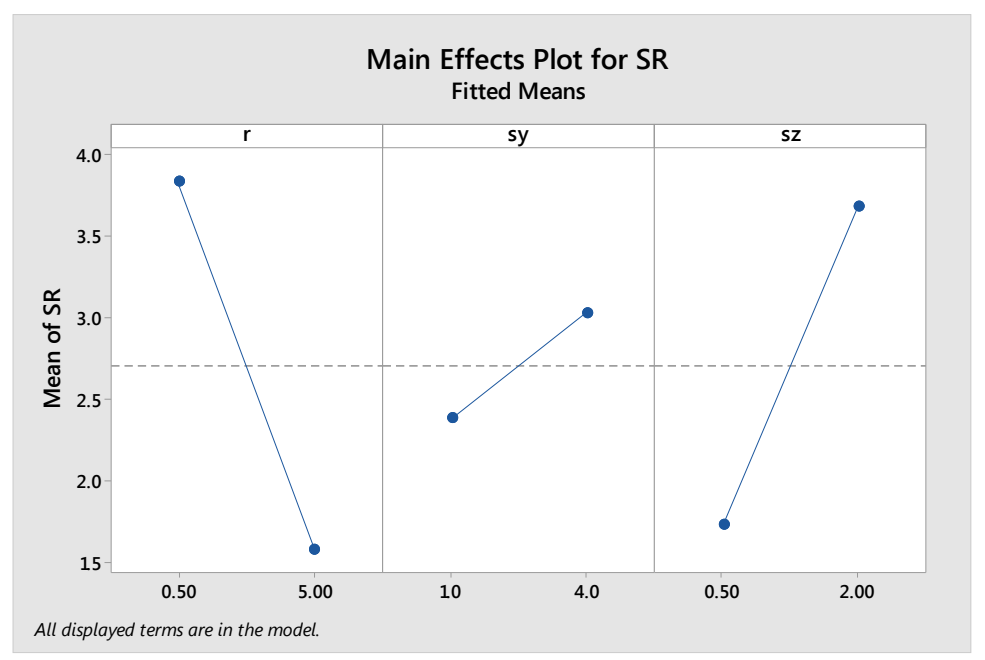

Fig 10. Effects plot for SR (set 2)

Another emotional surface quality that shows interactions is CF (Fig 11). Increased $r$ and $s y$ can both make surfaces feel formal, and the effect could be amplified in a synergistic manner if the two parameters are used together.

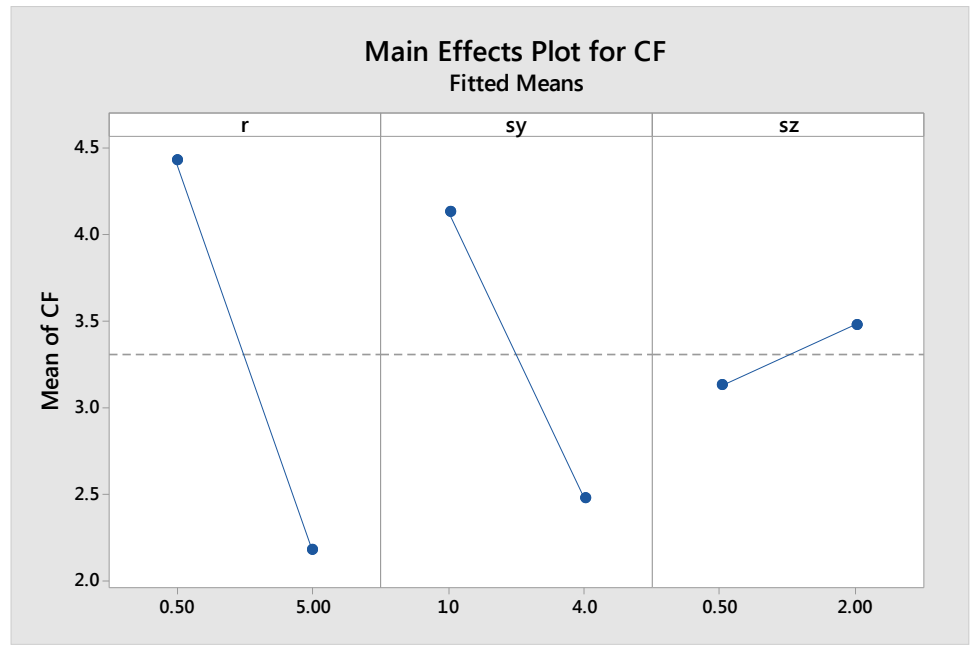

Fig 11. Effects plot for CF (set 2)

Interactions can take place in a different way. In case of MT (Fig 12), although none of the parameters were identified as significant ones, the interactions form a significant effect $(p=0.037)$ and this suggests that surfaces that evoke either modern or traditional feel, even to a certain extent only, could be designed by carefully combining those parameters. 


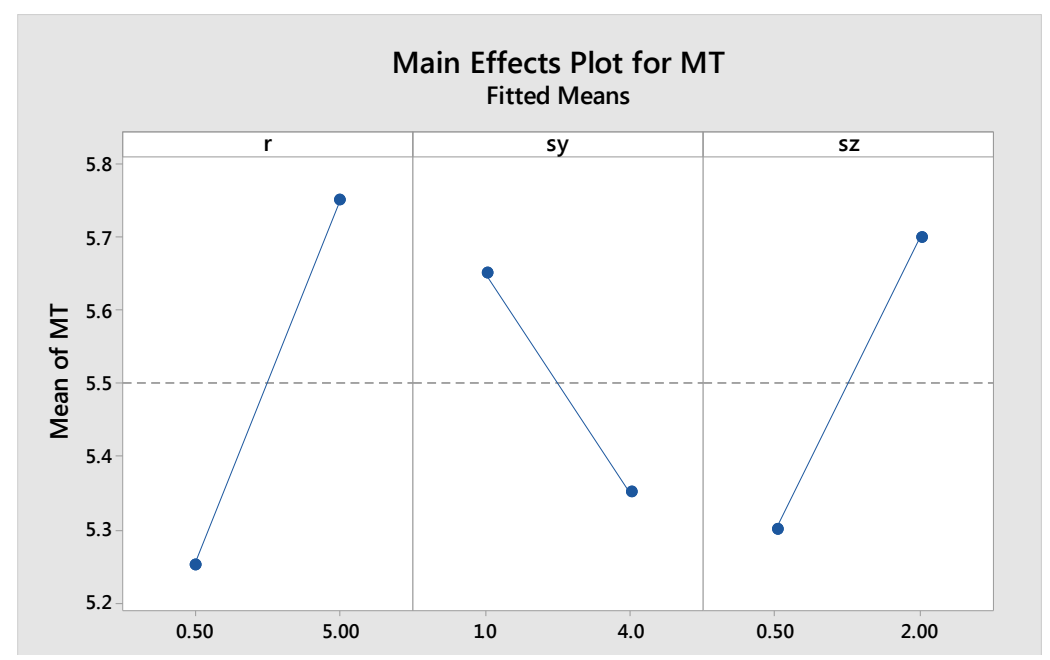

Fig 12. Effects plot for MT (set 2)

Figs 13 through 16 depict emotional qualities that $r$ is the sole parameter that has significant effect. Increased diameters are responsible for surfaces that feel warm (WC), simple (SB), sturdy (FS, against fragile), and blunt (SB), in order of significance, which is consistent with common experiences.

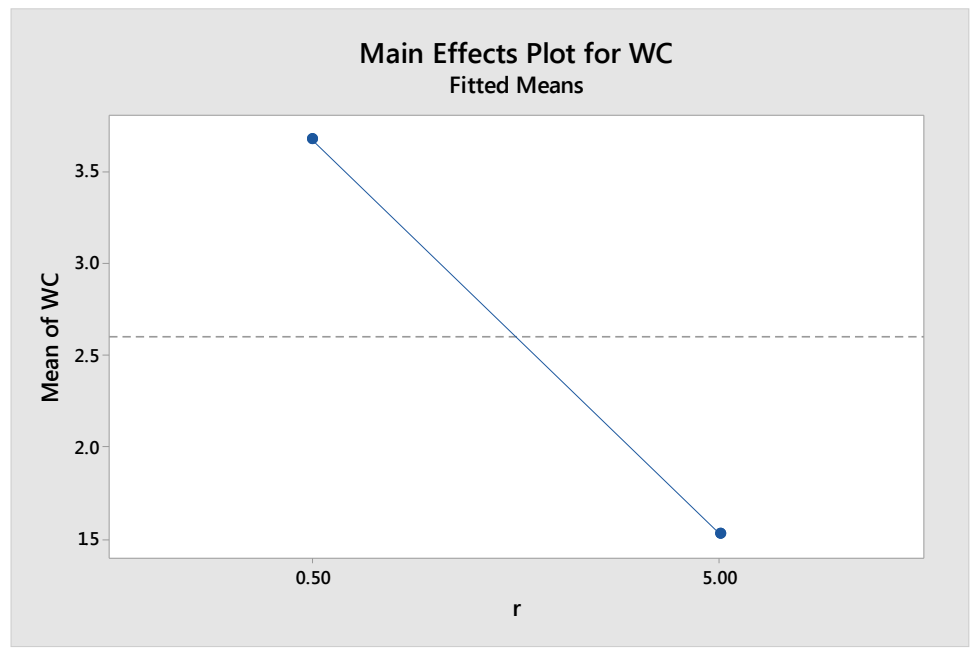

Fig 13. Effects plot for WC (set 2) 


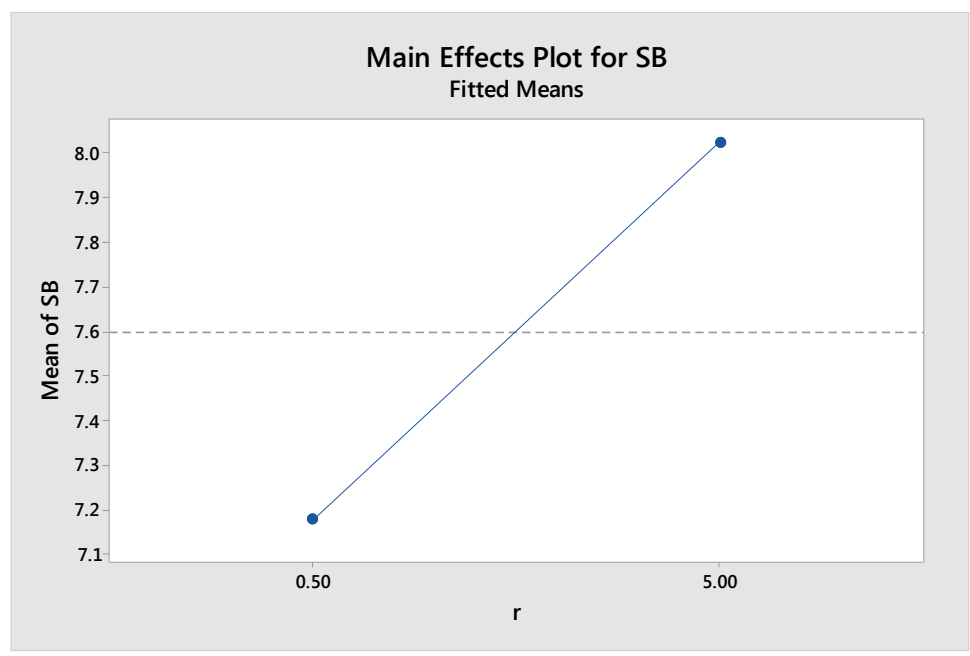

Fig 14. Effects plot for SB (set 2)

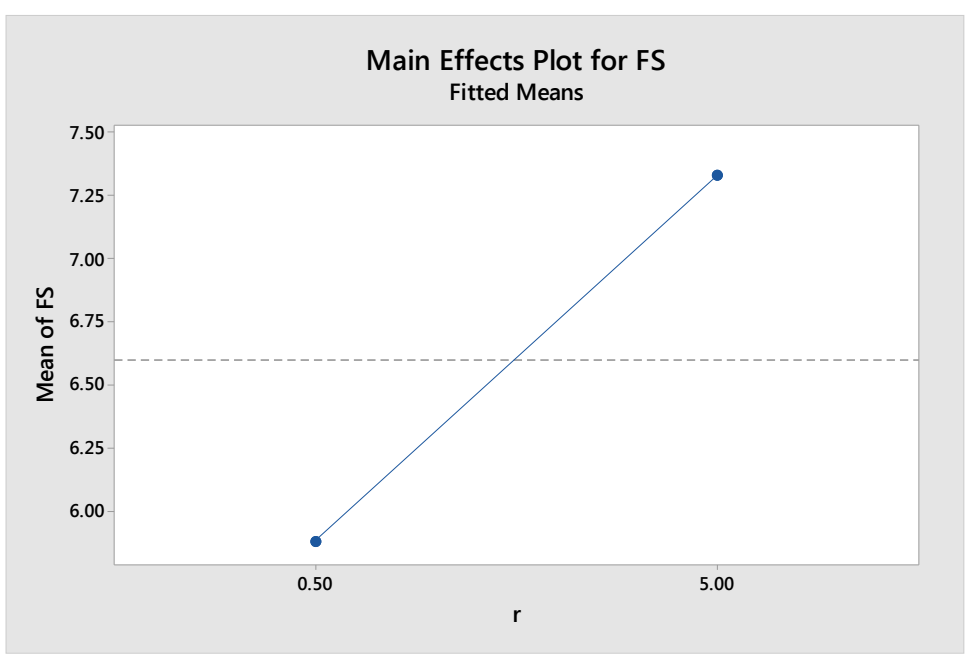

Fig 15. Effects plot for FS (set 2)

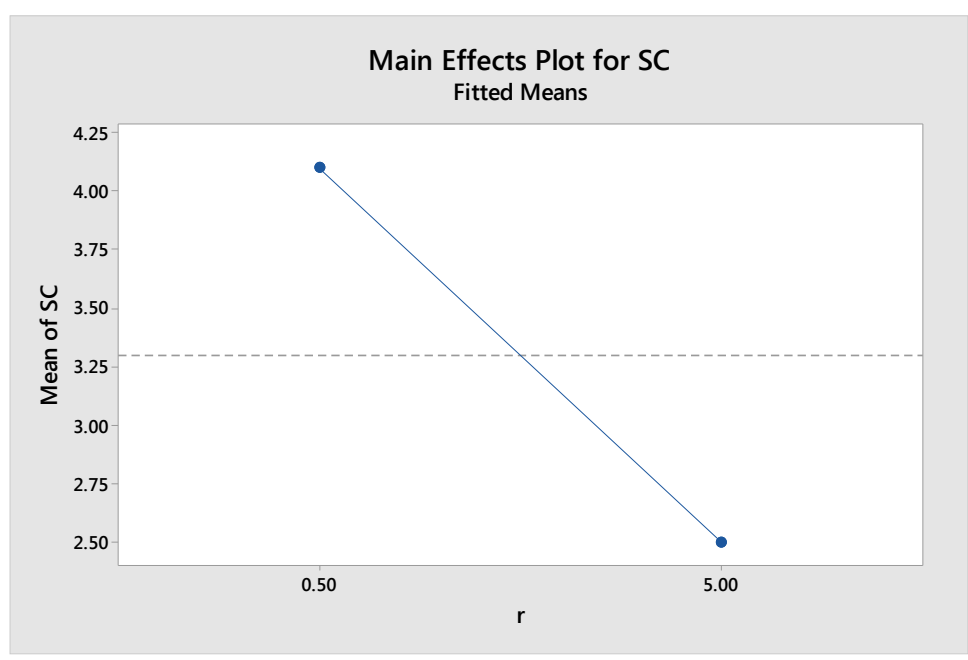

Fig 16. Effects plot for SC (set 2) 
Features with high aspect ratios along the Z-axis ( $s z)$ have significant effects on making the surfaces unattractive (AU), synthetic (NS), and unpleasant (PU, in order of significance), and the results in unison imply that the parameter may cause somewhat negative influence on design (Fig 17-19) unless used carefully.

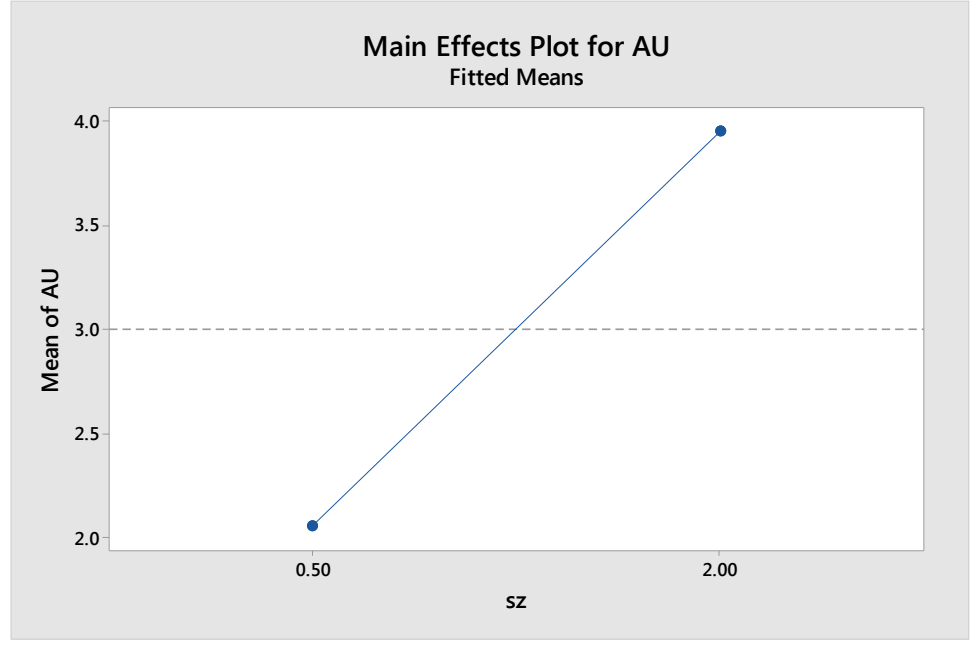

Fig 17. Effects plot for AU (set 2)

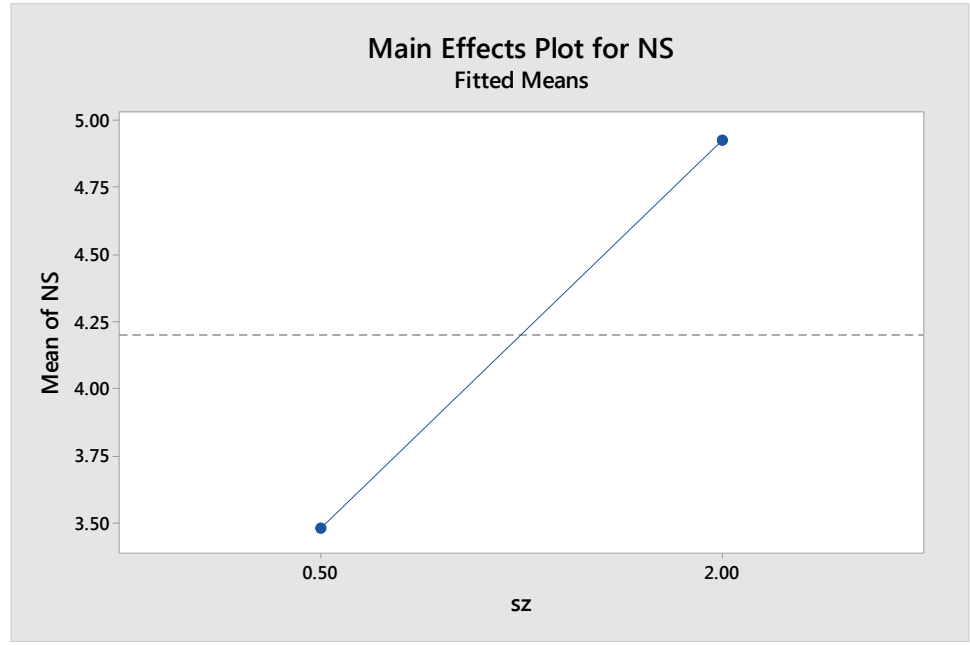

Fig 18. Effects plot for NS (set 2) 


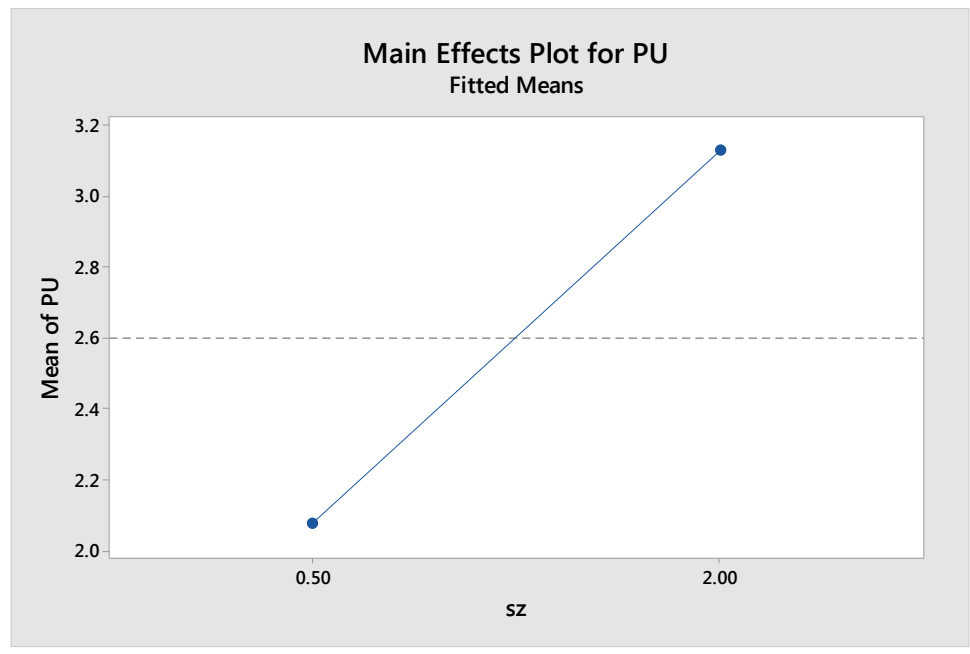

Fig 19. Effects plot for PU (set 2)

In set 3, various interactions among the parameters were observed. Figs 20 show one of the most complicated relationships in this research, which all parameters except for one (riy) have significant effects on the SB scale, individually or in combination with another. While higher proximity to the adjacent feature along Y-axis (py) plays the most significant role in making the surfaces feel sharp, probably by clearly revealing each sparsely distributed feature, degree of rotation of the features towards the X-axis (rux) has also significant effects, followed by length of a box along the $\mathrm{Y}$-axis $(f y)$, but in an inverse proportion to the former two. One remarkable factor is a combination of $f y$ and $m i$ as depicted in Fig 21. While $m i$, which refers to iteration steps within a unit distance, does not constitute any significant effect itself, it amplifies its impact when used in combination with $f y$, which means long box features placed at an increased interval may look blunt.

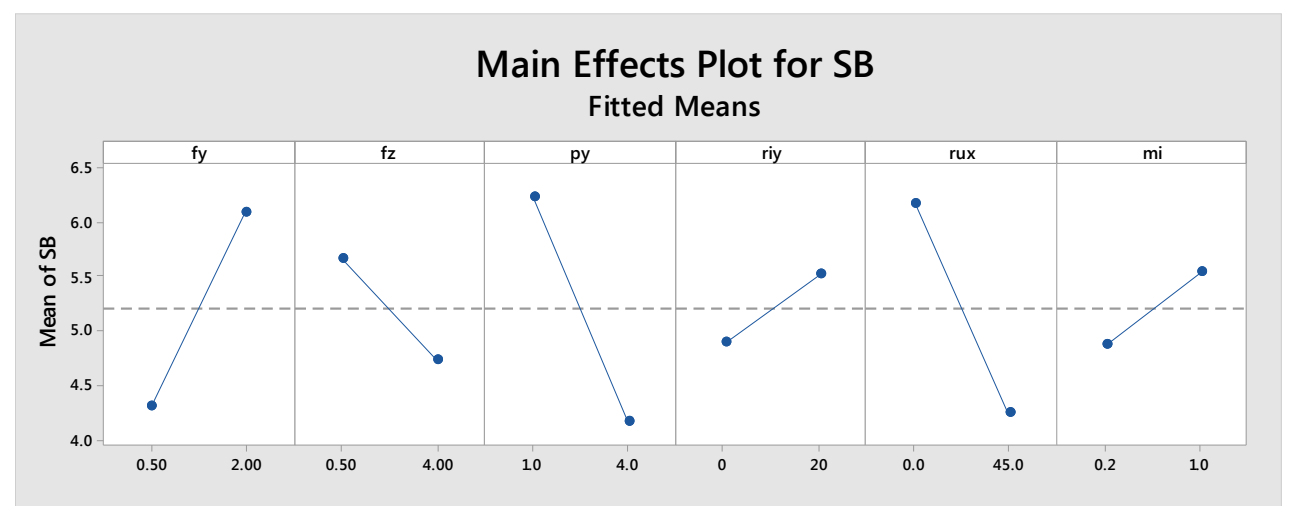

Fig 20. Effects plot for SB (set 3) 


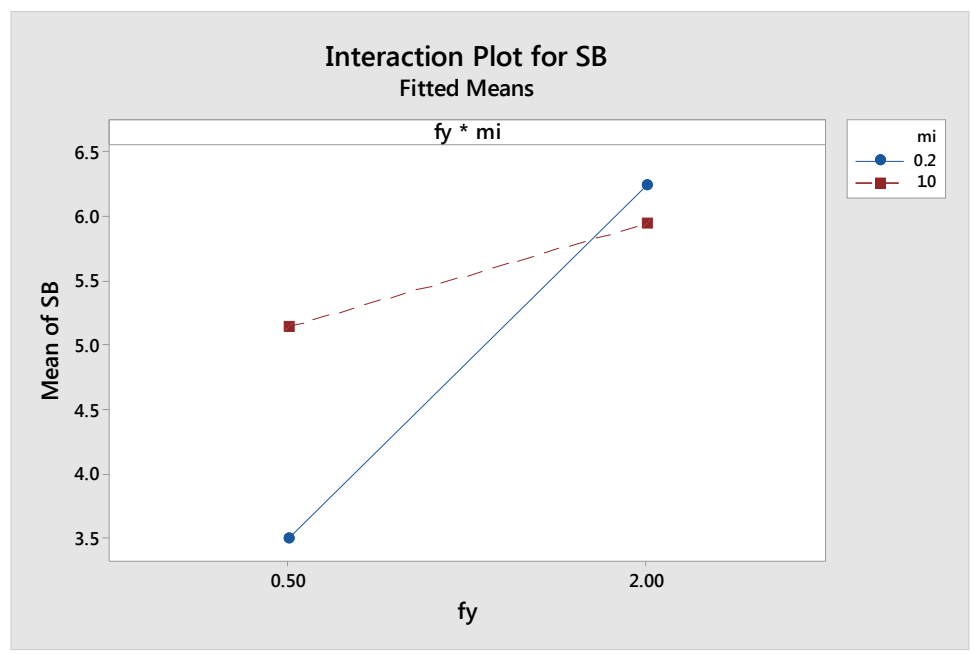

Fig 21. Interaction plot for $S B$ (set 3)

The combination has also significant effects on other emotional qualities including SR (Fig. 22), SC (Fig. 23), and WC (Fig. 24), and the latter two show a different type of interaction, one of which has a negative slope while the other has a positive one, even though the effect of $m i$ is considerably smaller than that of $f y$.

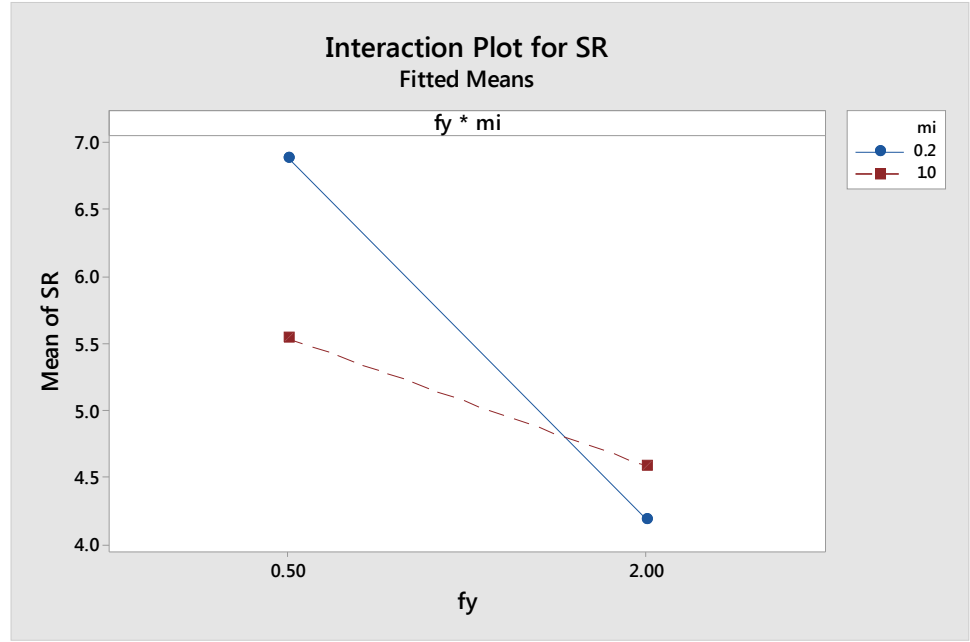

Fig 22. Interaction plot for SR (set 3$)$ 


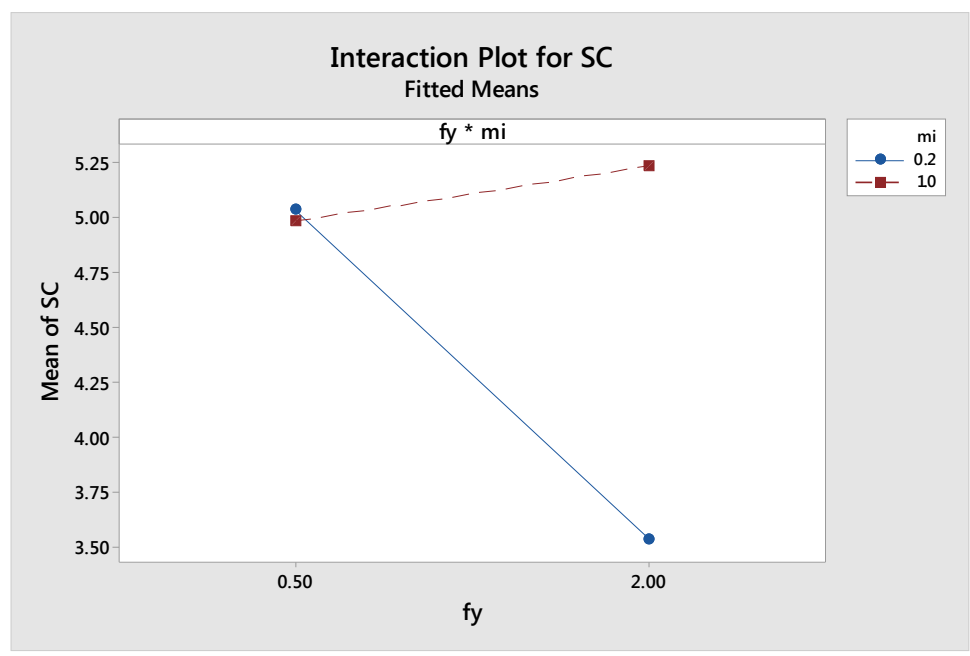

Fig 23. Interaction plot for SC (set 3)

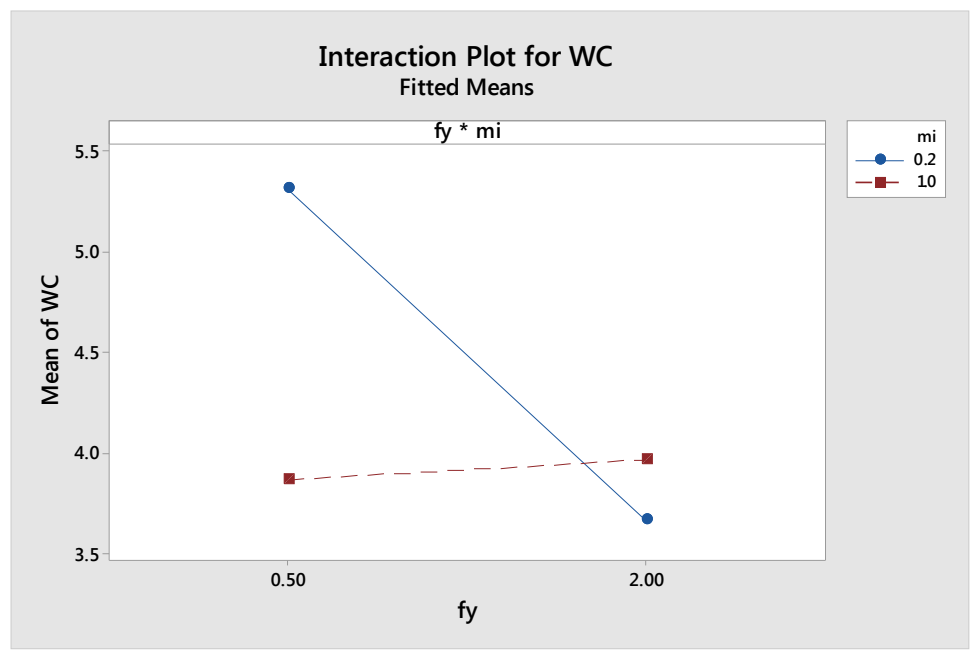

Fig 24. Interaction plot for WC (set 3)

Another interesting observation is that riy was identified as the sole parameter that has significant effect on $\mathrm{VC}$ scale (Fig 25), which hints that highly rotated boxes along the $\mathrm{Y}$-axis contribute to making the surfaces feel valuable. It is considered that the rotation reveals more facets of the boxes, possibly resulting an association with typical appearance of precious items such as diamond. 


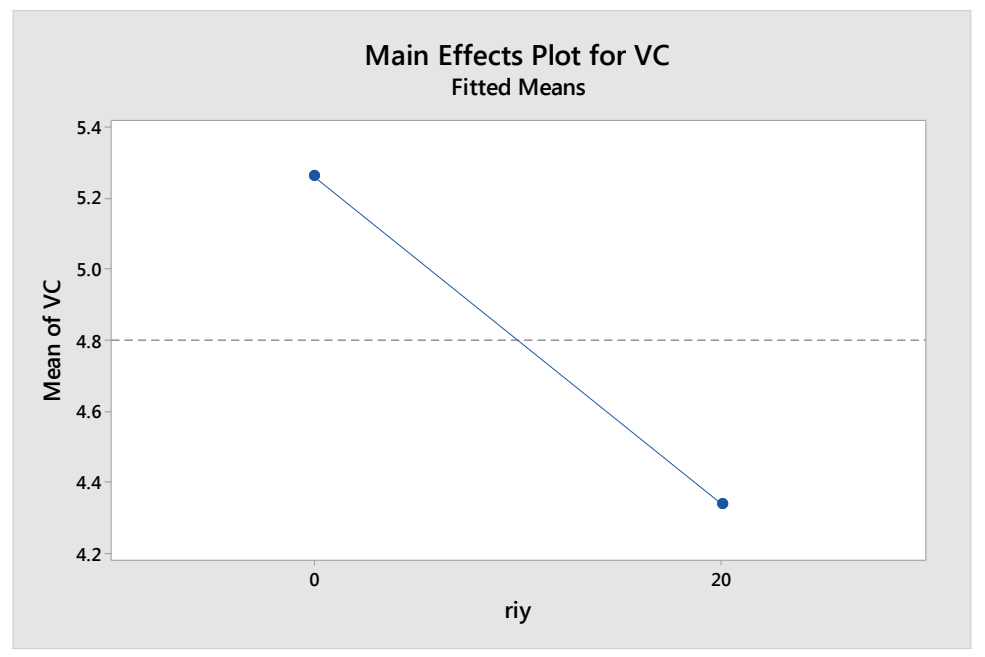

Fig 25. Interaction plot for VC (set 3)

Across the nine surfaces in set 3 , length of box features along the Z-axis $(f z)$ turned out to be the least influential parameter. It is quite different from the significant effects of $s z$, which dictates elongation of spheroid along the Z-axis observed in sets 1 and 2 . The different geometries of the individual features can be considered part of the reason, which needs further examination.

\section{Conclusion}

A wholly digital design process that includes planning of experiments, systematic arrangement of design parameters, preparation and production of objects, and analysis of user testing was attempted in order to investigate whether parameters could be explored and deliberately employed as a core element of emotive surfaces which evoke desired impressions and feelings through visual and tactile stimulation. A series of surface parameters as well as their effects on various emotional qualities in different levels were identified and quantitatively analysed. The experiments were conducted in three sets, each of which constitutes a development stage. It was confirmed in the first stage that the surfaces designed by combining discrete parameters instigated clearly recognisable emotional responses from participants. The second stage then proved that parameters can be used for multiple purposes across a range of emotional qualities, and the final stage demonstrated more complex utilisation of parameters especially interactions towards analytical synthesis of emotive surfaces which would otherwise not be possible. The process showed potential for purpose-specific, context-oriented, and user-focused creation of aesthetic yet functional surfaces.

\section{Acknowledgements}

This research was co-funded by Viclink (from Kiwinet) and New Zealand Product Accelerator.

\section{References}

DAGMAN, J.; KARLSSON, M.; WIKSTROM, L. (2010). "Investigating the haptic aspects of verbalised product experiences". International Journal of Design, Vol. 4 (3), pp.15-27.

HOPE, D., JONES, M., and ZUO, H. (2013). "Sensory perception in materials selection for industrial/product design" in The International Journal of Designed Objects, Vol. 6, No. 3, p. 17-31. 
JANSSON-BOYD, C. (2011). "Touch matters: exploring the relationship between consumption and tactile interaction" in Social Semiotics, Vol. 21, No. 4, p. 531-546.

KARANA, E., HEKKERT, P., and KANDACHAR, P. (2008). "Meanings of materials through sensorial properties and manufacturing processes" in Materials and Design, Vol. 30, p. 2778-2784.

KARANA, E., HEKKERT, P., and KANDACHAR, P. (2009). “A tool for meaning driven materials selection” in Materials and Design, Vol. 31, p. 2932-2941.

OUM, R. E., LIEBERMAN, D., and AYLWARD, A. (2011). "A feel for disgust: Tactile cues to pathogen presence" in Cognition and Emotion, Vol. 25, No.4, p. 717-725.

OXMAN, N. and ROSENBERG, J. L. (2007). "Material-based Design Computation: An Inquiry into Digital Simulation of Physical Material Properties as Design Generators" in International Journal of Architectural Computing (IJAC), Vol. 5, No. 1, p. 26-44.

PETKOVA, V. I.; ZETTERBERG, H.; EHRSSON, H. H. (2012). "Rubber Hands Feel Touch, but Not in Blind Individuals". PLoS ONE, Vol. 7 (4), e35912. < http://dx.doi.org/10.1371/journal.pone.0035912>[Accessed 25 Feb 2016]

PIAGET, J. (1952). The origins of intelligence in children. New York: International University Press.

TAKAHASHI, C.; DIEDRICHSEN, J.; WATT, S. J. (2009). "Integration of vision and haptics during tool use". Vision, Vol. 9 (6), pp.1-13.

WOHLERS, T, T. (2009). "Direct Digital Manufacturing” in Manufacturing Engineering, Vol. 142, No. 1, p.73 